\title{
Recurrence Relation and Accurate Value on Inverse Moment of Discrete Distributions
}

\author{
ChunYuan Wang and Wuyungaowa \\ Department of Mathematics, College of Sciences and Technology, Inner Mongolia University, Hohhot 010021, China
}

Correspondence should be addressed to ChunYuan Wang; wangchunyuan2006@163.com

Received 6 March 2015; Accepted 7 June 2015

Academic Editor: Zacharias Psaradakis

Copyright ( 92015 C. Wang and Wuyungaowa. This is an open access article distributed under the Creative Commons Attribution License, which permits unrestricted use, distribution, and reproduction in any medium, provided the original work is properly cited.

Properties of the generalized hypergeometric series functions are employed to get the recurrence relation for inverse moments and inverse factorial moments of some discrete distributions. Meanwhile, with the existence of the recurrence relations, the accurate value for inverse moment of discrete distributions can thus be obtained.

\section{Introduction and Preliminaries}

Kumar and Consul [1] develop a recursive relation upon the negative moments of power series distribution. The recurrence relation for the negative moments of the Poisson distribution was first derived by Chao and Strawderman [2], after which it is shown by Kumar and Consul [1] as a special case of their result. Using the recurrence relation for the negative moments of the Lagrangian binomial distribution, Kumar and Consul [1] have established the binomial and negative binomial distributions.

Ahmad and Saboor [3] proved many properties of the hypergeometric series. Besides, the following series have been provided by Ahmad and Saboor [3] and

$$
\begin{aligned}
& p_{p} F_{q}\left[\left(a_{1}, k\right),\left(a_{2}, k\right), \ldots,\left(a_{p}, k\right) ;\left(b_{1}, k\right),\left(b_{2}, k\right), \ldots,\right. \\
& \left.\left(b_{q}, k\right) ; z\right]=1+\frac{a_{1}^{k} \cdot a_{2}^{k} \cdots a_{p}^{k}}{b_{1}^{k} \cdot b_{2}^{k} \cdots b_{q}^{k}} z \\
& +\frac{\left[a_{1}\left(a_{1}+1\right)\right]^{k}\left[a_{2}\left(a_{2}+1\right)\right]^{k} \cdots\left[a_{p}\left(a_{p}+1\right)\right]^{k}}{\left[b_{1}\left(b_{1}+1\right)\right]^{k}\left[b_{2}\left(b_{2}+1\right)\right]^{k} \cdots\left[b_{q}\left(b_{q}+1\right)\right]^{k}} \frac{z^{2}}{2 !} \\
& +\cdots
\end{aligned}
$$

is generalized hypergeometric series, where $\left(\lambda_{i}^{k}\right)_{n}=\left(\lambda_{i}^{k}\right)\left(\lambda_{i}+\right.$ $1)^{k}\left(\lambda_{i}+2\right)^{k} \cdots\left(\lambda_{i}+n-1\right)^{k},\left(\lambda_{i}^{k}\right)_{0}=1, i=1,2, \ldots, p, k=$ $1,2, \ldots$. If $k=1$, then

$$
{ }_{p} F_{q}=\sum_{n=0}^{\infty} \frac{\left(a_{1}\right)_{n}\left(a_{2}\right)_{n} \cdots\left(a_{p}\right)_{n}}{\left(b_{1}\right)_{n}\left(b_{2}\right)_{n} \cdots\left(b_{q}\right)_{n}} \frac{z^{n}}{n !}
$$

is hypergeometric series.

In this paper, the recurrence relation for negative moments along with negative factorial moments of some discrete distributions can be obtained. These relations have been derived with properties of the hypergeometric series.

In the next part, some necessary definitions have been introduced.

Let $X$ be a generalized negative binomially distributed random variable with parameters $\theta, \beta$ and the probability mass function is

$$
\begin{array}{r}
P_{x}(\theta, \beta)=\frac{m}{m+\beta x}\left(\begin{array}{c}
m+\beta x \\
x
\end{array}\right) \theta^{x}(1-\theta)^{m+\beta x-x}, \\
x=0,1,2, \ldots,
\end{array}
$$

where $0<\theta<1,|\theta \beta|<1,1 \leq \beta \leq \theta^{-1}$, and $m>0$ and a constant. 
Let $X$ be a generalized Poisson distribution with parameters $\lambda, \theta$ and the probability mass function is

$$
P_{x}(\theta)=e^{-\lambda(1+\theta x)} \frac{\lambda^{x}(1+\theta x)^{x-1}}{x !}, \quad x=0,1, \ldots
$$

where $0<\lambda<\infty,|\lambda \theta|<1$, and $0<\theta<1$.

Let the random variable $X$ be equipped with a generalized poisson-negative-binomial distribution with parameters $\lambda, \theta$, and $\beta$; the probability mass function is

$$
\begin{aligned}
& P_{x}(\theta, \beta) \\
& \quad=e^{-\lambda(1+\theta s)} \sum_{s=0}^{\infty} \frac{\lambda^{s(1+\theta s)^{s-1}}}{s !} \frac{m}{m+\beta x}\left(\begin{array}{c}
m+\beta x \\
x
\end{array}\right) \theta^{x}(1 \\
& -\theta)^{m+\beta x-x}, \quad x=0,1,2, \ldots,
\end{aligned}
$$

where $0<\theta<1,0<\lambda<\infty,|\lambda \theta|<1,|\theta \beta|<1,1 \leq \beta \leq \theta^{-1}$, $s \geq 0, s \in \mathbf{Z}$, and $m>0$ and a constant.

Let $X$ be a generalized logarithmic distributed random variable with parameters $\theta$ and $\beta$; the probability mass function is

$$
P_{x}(\theta, \beta)=\frac{1}{\beta x}\left(\begin{array}{c}
\beta x \\
x
\end{array}\right) \frac{\theta^{x}(1-\theta)^{\beta x-x}}{[-\log (1-\theta)]}, \quad x=1,2, \ldots,
$$

where $0<\theta<1,0<\theta \beta<1$, and $\beta \geq 1$.

\section{The Recurrence Relation for Inverse Moments of Some Discrete Distributions}

In this section, some recurrence relations for inverse moments of some discrete distributions can be obtained with the properties of the generalized hypergeometric series functions.

Theorem 1. Let $X$ be a generalized negative binomial random variable with parameters $\theta, \beta$, for $0<\theta<1,|\theta \beta|<1,1 \leq \beta \leq$ $\theta^{-1}$, and probability mass function is defined in (3), and then the inverse moment of first order is given by

$$
\begin{aligned}
& E(X+A)^{-1}=\frac{m \theta(1-\theta)^{m+\beta-1}}{A+1}{ }_{3} F_{2}[A+1, m \\
& \left.\quad+(n+1) \beta-n, 1 ; A+2,2 ; \frac{\theta}{(1-\theta)^{1-\beta}}\right],
\end{aligned}
$$

where $A \geq 0, n=0,1,2, \ldots$, and $m>0$ and a constant.
Proof. Since $X$ is a generalized negative binomial random variable with parameters $\theta, \beta$, then

$$
\begin{aligned}
E & (X+A)^{-1}=\sum_{x=1}^{\infty} \frac{m}{m+\beta x}\left(\begin{array}{c}
m+\beta x \\
x
\end{array}\right) \theta^{x}(1-\theta)^{m+\beta x-x} \frac{1}{x+A} \\
& =\frac{m \theta(1-\theta)^{m+\beta-1}}{A+1}\left\{1+\frac{(A+1)(m+2 \beta-1)}{(A+2) \cdot 2 !} \frac{\theta}{(1-\theta)^{1-\beta}}\right. \\
& \left.+\frac{(A+1)(m+3 \beta-1)(m+3 \beta-2)}{(A+3) \cdot 3 !} \frac{\theta^{2}}{(1-\theta)^{2(1-\beta)}}+\cdots\right\} \\
& =\frac{m \theta(1-\theta)^{m+\beta-1}}{(A+1)}\left\{1+\frac{(A+1)(m+2 \beta-1) \cdot 1}{(A+2) \cdot 2 \cdot 1 !} \frac{\theta}{(1-\theta)^{1-\beta}}\right. \\
& +\frac{(A+1)(A+2)(m+3 \beta-1)(m+3 \beta-2) \cdot 1 \cdot 2}{(A+2)(A+3) \cdot 2 \cdot 3 \cdot 2 !}\left[\frac{\theta}{(1-\theta)^{1-\beta}}\right]^{2} \\
& +\cdots\}=\frac{m \theta(1-\theta)^{m+\beta-1}}{(A+1)}{ }_{3} F_{2}[A+1, m+(n+1) \beta-n, 1 ; A \\
& \left.+2,2 ; \frac{\theta}{(1-\theta)^{1-\beta}}\right] .
\end{aligned}
$$

Theorem 2. Let $X$ be a generalized negative binomially distributed random variable with parameters $\theta$ and $\beta$, and probability mass function is defined in (3). Then the following relation holds:

$$
\begin{aligned}
A z & (p-A-1) E(X+A)^{-1} \\
& =A(1-A) E(X+A-1)^{-1}-P_{1}(1-z)^{-p+1}+Q_{0},
\end{aligned}
$$

where $Q_{0}=[A+(p-A-1) z]_{2} F_{1}[1, p ; 2 ; z], p=m+(n+1) \beta-n$, $n=0,1,2, \ldots, z=\theta /(1-\theta)^{1-\beta}$, and $P_{1}=P(X=1)=$ $m \theta(1-\theta)^{m+\beta+1}$.

Proof. From Theorem 1, we have

$$
E(X+A)^{-1}=\frac{P_{1}}{A+1}{ }_{3} F_{2}[A+1, p, 1 ; A+2,2 ; z],
$$

using the identity (see [4, page 85$]$ )

$$
\begin{aligned}
& (1-x){ }_{3} F_{2}\left[\alpha_{1}, \alpha_{2}, \alpha_{3} ; \beta_{1}, \beta_{2} ; x\right] \\
& ={ }_{3} F_{2}\left[\alpha_{1}-1, \alpha_{2}, \alpha_{3} ; \beta_{1}, \beta_{2} ; x\right]+x \\
& \quad \cdot \frac{\left(\alpha_{2}-\beta_{1}\right)\left(\alpha_{3}-\beta_{1}\right)}{\beta_{1}\left(\beta_{2}-\beta_{1}\right)}{ }_{3} F_{2}\left[\alpha_{1}, \alpha_{2}, \alpha_{3} ; \beta_{1}+1, \beta_{2} ; x\right] \\
& +x \frac{\left(\alpha_{2}-\beta_{2}\right)\left(\alpha_{3}-\beta_{2}\right)}{\beta_{2}\left(\beta_{1}-\beta_{2}\right)} \\
& \cdot{ }_{3} F_{2}\left[\alpha_{1}, \alpha_{2}, \alpha_{3} ; \beta_{1}, \beta_{2}+1 ; x\right] .
\end{aligned}
$$


For $\alpha_{1}=A+1, \alpha_{2}=p, \alpha_{3}=1, \beta_{1}=2, \beta_{2}=A+1$, and $x=z$ we have

$$
\begin{aligned}
& (1-z){ }_{3} F_{2}[A+1, p, 1 ; A+1,2 ; z] \\
& ={ }_{3} F_{2}[A, p, 1 ; A+1,2 ; z] \\
& \quad+\frac{(2-p) z}{2(A-1)}{ }_{3} F_{2}[A+1, p, 1 ; A+1,3 ; z] \\
& \quad+\frac{A z(p-A-1)}{A^{2}-1}{ }_{3} F_{2}[A+1, p, 1 ; A+2,2 ; z],
\end{aligned}
$$

and we know that

$$
\begin{aligned}
& { }_{3} F_{2}[A+1, p, 1 ; A+1,2 ; z]={ }_{2} F_{1}[1, p ; 2 ; z], \\
& { }_{3} F_{2}[A+1, p, 1 ; A+1,3 ; z]={ }_{2} F_{1}[1, p ; 3 ; z] .
\end{aligned}
$$

Then (12) becomes

$$
\begin{aligned}
& (1-z){ }_{2} F_{1}[1, p ; 2 ; z] \\
& ={ }_{3} F_{2}[A, p, 1 ; A+1,2 ; z] \\
& \quad+\frac{(2-p) z}{2(A-1)}{ }_{2} F_{1}[1, p ; 3 ; z] \\
& \quad+\frac{A z(p-A-1)}{A^{2}-1}{ }_{3} F_{2}[A+1, p, 1 ; A+2,2 ; z] .
\end{aligned}
$$

Using another identity (see [4, page 71$]$ )

$$
\begin{aligned}
& {[a+(b-c) z]{ }_{2} F_{1}[a, b ; c ; z] } \\
&= a(1-z){ }_{2} F_{1}[a+1, b ; c ; z] \\
& \quad-\frac{(c-a)(c-b) z}{c}{ }_{2} F_{1}[a, b ; c+1 ; z],
\end{aligned}
$$

and for $a=1, b=p, c=2$, and $z=z$ we have

$$
\begin{aligned}
& {[1+(p-2) z]_{2} F_{1}[1, p ; 2 ; z]} \\
& \quad=(1-z){ }_{2} F_{1}[2, p ; 2 ; z]-\frac{(2-p) z}{2}{ }_{2} F_{1}[1, p ; 3 ; z]
\end{aligned}
$$

as

$$
{ }_{2} F_{1}[2, p ; 2 ; z]={ }_{1} F_{0}[p ;-; z]=(1-z)^{-p} \text {. }
$$

From (14) and (16) we have

$$
\begin{aligned}
(1-z){ }_{2} F_{1}[1, p ; 2 ; z] & \\
= & { }_{3} F_{2}[A, p, 1 ; A+1,2 ; z] \\
& +\frac{A z(p-A-1)}{A^{2}-1}{ }_{3} F_{2}[A+1, p, 1 ; A+2,2 ; z] \\
& -\frac{1+(p-2) z}{A-1}{ }_{2} F_{1}[1, p ; 2 ; z]+\frac{(1-z)^{-p+1}}{A-1} .
\end{aligned}
$$

Rearranging we get

$$
\begin{gathered}
\frac{A+(p-A-1) z}{A-1}{ }_{2} F_{1}[1, p ; 2 ; z]-\frac{(1-z)^{-p+1}}{A-1} \\
=\frac{A}{P_{1}} E(X+A-1)^{-1} \\
+\frac{A z(p-A-1)}{P_{1}(A-1)} E(X+A)^{-1} .
\end{gathered}
$$

By collating, we get the result (9).

Theorem 3. Let the random variable $X$ be equipped with a generalized Poisson distribution with parameters $\theta$ and $\lambda$, and probability mass function is defined in (4). Then

$$
\begin{aligned}
A z E(X+A)^{-1}= & A(1-A) E(X+A-1)^{-1} \\
& +A P_{1}{ }_{1} F_{1}[1 ; 2 ; z]-P_{1},
\end{aligned}
$$

and $P_{1}=P(X=1)=\lambda e^{-\lambda(1+\theta)}, z=\lambda(1+(n+1) \theta) / e^{\lambda \theta}$, and $n=0,1,2, \ldots$.

Proof. Using the identity (see [4, page 84$]$ )

$$
\begin{aligned}
& { }_{2} F_{2}\left[\alpha_{1}, \alpha_{2} ; \beta_{1}, \beta_{2} ; x\right] \\
& ={ }_{2} F_{2}\left[\alpha_{1}-1, \alpha_{2} ; \beta_{1}, \beta_{2} ; x\right] \\
& \quad+x \frac{\alpha_{2}-\beta_{1}}{\beta_{1}\left(\beta_{2}-\beta_{1}\right)}{ }_{2} F_{2}\left[\alpha_{1}, \alpha_{2} ; \beta_{1}+1, \beta_{2} ; x\right] \\
& \quad+x \frac{\alpha_{2}-\beta_{2}}{\beta_{2}\left(\beta_{1}-\beta_{2}\right)}{ }_{2} F_{2}\left[\alpha_{1}, \alpha_{2} ; \beta_{1}, \beta_{2}+1 ; x\right] .
\end{aligned}
$$

By the same as Theorem 2 calculating, we get the result (20).

Theorem 4. Let the random variable $X$ be equipped with a generalized Poisson-negative-binomial distribution with parameters $\lambda, \theta$, and $\beta$, and probability mass function is defined in (5); then

$$
\begin{aligned}
A z & (p-A-1) E(X+A)^{-1} \\
= & A(1-A) E(X+A-1)^{-1}-P_{1}(1-z)^{-p+1} \\
& +Q_{1},
\end{aligned}
$$

where $P_{1}=P(X=1)=e^{-\lambda(1+\theta s)} \sum_{s=0}^{\infty}\left(\lambda^{s}(1+\theta s)^{s-1} / s !\right) m \theta(1-$ $\theta)^{m+\beta-1}, p=m+(n+1) \beta-n, n=0,1,2, \ldots, Q_{1}=P_{1}[A+$ $(p-A-1) z{ }_{2} F_{1}[1 ; p ; 2 ; z]$, and $z=\theta /(1-\theta)^{1-\beta}$.

Proof. Using identities (11) and (15), by the same as Theorem 2 calculating, we get the result (22).

Theorem 5. Let $X$ be a generalized logarithmic distributed random variable with parameters $\theta$ and $\beta$, and probability 
mass function is defined in (6). Then the following recurrence relation holds:

$$
\begin{aligned}
A z( & (p-A-1) E(X+A)^{-1} \\
= & A(1-A) E(X+A-1)^{-1}-P_{1}(1-z)^{-p+1} \\
& +Q_{2},
\end{aligned}
$$

where $P_{1}=P(X=1)=\theta(1-\theta)^{\beta-1} /[-\log (1-\theta)], Q_{2}=P_{1}[A+$ $(p-A-1) z]_{2} F_{1}[1, p ; 2 ; z], p=(n+1) \beta-n, n=0,1,2, \ldots$, and $z=\theta /(1-\theta)^{1-\beta}$.

Proof. Using identities (11) and (15), by the same as Theorem 2 calculating, we get the result (23).

\section{Recurrence Relation for Inverse Factorial Moments of Discrete Distributions}

In this section, some recurrence relations for inverse factorial moments of some discrete distributions can be obtained with the properties of the generalized hypergeometric series functions.

Theorem 6. Let $X$ be a generalized negative binomial random variable with parameters $\theta, \beta$, for $0<\theta<1,|\theta \beta|<1,1 \leq \beta \leq$ $\theta^{-1}$, and probability mass function is defined in (3), and then the factorial inverse moment of first order is given by

$$
\begin{aligned}
& E\left[\prod_{i=1}^{l}(x+i)\right]^{-1}=\frac{m \theta(1-\theta)^{m+\beta-1}}{(l+1) !}{ }_{3} F_{2}[2, m \\
& \left.\quad+(n+1) \beta-n, 1 ; l+2,2 ; \frac{\theta}{(1-\theta)^{1-\beta}}\right],
\end{aligned}
$$

where $l \geq 1$ and $l \in \mathbf{Z}^{+}, n=0,1,2, \ldots$.

Proof. Since $X$ is a generalized negative binomial random variable with parameters $\theta, \beta$, then

$$
\begin{aligned}
E[ & \left.\prod_{i=1}^{l}(x+i)\right]^{-1} \\
& =\sum_{x=1}^{l} \frac{m}{m+\beta x}\left(\begin{array}{c}
m+\beta x \\
x
\end{array}\right) \theta^{x}(1-\theta)^{m+\beta x-x} \prod_{i=1}^{l}\left(\frac{1}{x+i}\right) \\
& =\sum_{x=1}^{l} \frac{m}{m+\beta x}\left(\begin{array}{c}
m+\beta x \\
x
\end{array}\right) \theta^{x}(1-\theta)^{m+\beta x-x}\left[\frac{x !}{(l+x) !}\right] \\
& =\frac{m \theta(1-\theta)^{m+\beta-1}}{(l+1) !}\left\{\begin{array}{c}
1+\frac{2(m+2 \beta-1) \cdot 1}{(l+2) \cdot 2 \cdot 1 !} \frac{\theta}{(1-\theta)^{1-\beta}} \\
+
\end{array}\right. \\
& +\cdots\}=\frac{m \theta(1-\theta)^{m+\beta-1}}{(l+1) !}{ }_{2} F_{1}[m+(n+1) \beta-n, 1 ; l \\
& \left.+2 ; \frac{\theta}{(1-\theta)^{1-\beta}}\right]
\end{aligned}
$$

Theorem 7. Let $X$ equipped with generalized negative binomial probability distribution be defined in (3) with parameters $\theta$ and $\beta$; suppose $\mu_{-[l]}^{\prime}$ is the lth negative factorial moment of $X$. Then the relation

$$
\begin{aligned}
& (l+1)(p-l-2) z \mu_{-[l+1]}^{\prime} \\
& \quad=[(p-2 l-2) z+l+1] \mu_{-[l]}^{\prime}-(1-z) \mu_{-[l-1]}^{\prime}
\end{aligned}
$$

holds for $l=2,3,4, \ldots$, and $p=m+(n+1) \beta-n, n=0,1,2, \ldots$, $z=\theta /(1-\theta)^{1-\beta}$.

Proof. From Theorem 6, we have

$$
\mu_{-[l]}^{\prime}=\frac{m \theta(1-\theta)^{m+\beta-1}}{(l+1) !}{ }_{2} F_{1}[1, p ; l+2 ; z] .
$$

Using the identity (see [4, page 71$]$ )

$$
\begin{aligned}
& (a-c+1){ }_{2} F_{1}[a, b ; c ; z] \\
& \quad=a_{2} F_{1}[a+1, b ; c ; z]-(c-1){ }_{2} F_{1}[a, b ; c-1 ; z],
\end{aligned}
$$

and for $a=1, b=p, c=l+3$, and $z=z$ we have

$$
\begin{aligned}
{ }_{2} F_{1}[1, p ; l+3 ; z]= & \frac{l+2}{l+1}{ }_{2} F_{1}[1, p ; l+2 ; z] \\
& -\frac{1}{l+1}{ }_{2} F_{1}[2, p ; l+3 ; z] .
\end{aligned}
$$

Using the identity (see [4, page 71$]$ )

$$
\begin{aligned}
& (1-z){ }_{2} F_{1}\left[a_{1}, b_{1} ; c_{1} ; z\right] \\
& ={ }_{2} F_{1}\left[a_{1}-1, b_{1} ; c_{1} ; z\right] \\
& \quad-\frac{c_{1}-b_{1}}{c_{1}} z_{2} F_{1}\left[a_{1}, b_{1} ; c_{1}+1 ; z\right],
\end{aligned}
$$

for $a_{1}=2, b_{1}=p, c=l+2$, and $z=z$; rearranging we have

$$
\begin{aligned}
{ }_{2} F_{1} & {[2, p ; l+3 ; z] } \\
= & \frac{(l+2)(1-z)}{(p-l-2) z}{ }_{2} F_{1}[2, p ; l+2 ; z] \\
& -\frac{l+2}{(p-l-2) z}{ }_{2} F_{1}[1, p ; l+2 ; z] .
\end{aligned}
$$

Substituting in (29), we get

$$
\begin{aligned}
{ }_{2} F_{1} & {[1, p ; l+3 ; z] } \\
= & \frac{(l+2)[(p-l-2) z+1]}{(l+1)(p-l-2) z}{ }_{2} F_{1}[1, p ; l+2 ; z] \\
& -\frac{(l+2)(1-z)}{(l+1)(p-l-2) z}{ }_{2} F_{1}[2, p ; l+2 ; z] .
\end{aligned}
$$

Consider ${ }_{2} F_{1}[2, p ; l+2 ; z]$ and using (28), for $a=1, b=p$, $c=l+2$, and $z=z$ we have

$$
\begin{aligned}
{ }_{2} F_{1}[2, p ; l+2 ; z]= & (l+1){ }_{2} F_{1}[1, p ; l+1 ; z] \\
& -l_{2} F_{1}[1, p ; l+2 ; z] .
\end{aligned}
$$


Substituting in (32), we get

$$
\begin{aligned}
{ }_{2} F_{1}[ & 1, p ; l+3 ; z] \\
= & \frac{(l+2)[(p-l-2) z+1]}{(l+1)(p-l-2) z}{ }_{2} F_{1}[1, p ; l+2 ; z] \\
& -\frac{(l+2)(1-z)}{(p-l-2) z}{ }_{2} F_{1}[1, p ; l+1 ; z] \\
& +\frac{l(l+2)(1-z)}{(l+1)(p-l-2) z}{ }_{2} F_{1}[1, p ; l+2 ; z] \\
= & \frac{(l+2)[(p-2 l-2) z+l+1]}{(l+1)(p-l-2) z}{ }_{2} F_{1}[1, p ; l+2 ; z] \\
& -\frac{(l+2)(1-z)}{(p-l-2) z}{ }_{2} F_{1}[1, p ; l+1 ; z] .
\end{aligned}
$$

Rearranging we have

$$
\begin{aligned}
& \frac{(l+2) !}{m \theta(1-\theta)^{m+\beta-1}} \mu_{-[l+1]}^{\prime} \\
& =\frac{(l+2)[(p-2 l-2) z+l+1]}{(l+1)(p-l-2) z} \frac{(l+1) !}{m \theta(1-\theta)^{m+\beta-1}} \mu_{-[l]}^{\prime} \\
& \quad-\frac{(l+2)(1-z)}{(l+1)(p-l-2) z} \frac{(l+1) !}{m \theta(1-\theta)^{m+\beta-1}} \mu_{-[l-1]}^{\prime},
\end{aligned}
$$

and by collating, we get the result (26).

Theorem 8. Let the random variable $X$ equipped with a generalized Poisson distribution be defined in (4) with parameters $\lambda$ and $\theta$, and $\mu_{-[l]}^{\prime}$ is the lth negative factorial moment of $X$. Then the relation

$$
(l+1) z \mu_{-[l+1]}^{\prime}=(l+z+1) \mu_{-[l]}^{\prime}-\mu_{-[l-1]}^{\prime}
$$

holds for $l=2,3,4, \ldots, z=(1+(n+1) \theta) \lambda / e^{\lambda \theta}, \lambda, \theta>0$, $n=0,1,2, \ldots$

Proof. Using the identity (see [4, pages 82 and 84$]$ )

$$
\begin{aligned}
\left(\alpha_{1}-\right. & \left.\beta_{1}+1\right){ }_{2} F_{2}\left[\alpha_{1}, \alpha_{2} ; \beta_{1}, \beta_{2} ; x\right] \\
= & \alpha_{1}{ }_{2} F_{2}\left[\alpha_{1}+1, \alpha_{2} ; \beta_{1}, \beta_{2} ; x\right] \\
& \quad-\left(\beta_{1}-1\right){ }_{2} F_{2}\left[\alpha_{1}, \alpha_{2} ; \beta_{1}-1, \beta_{2} ; x\right], \\
{ }_{2} F_{2}[ & \left.\alpha_{1}, \alpha_{2} ; \beta_{1}, \beta_{2} ; x\right] \\
= & { }_{2} F_{2}\left[\alpha_{1}-1, \alpha_{2} ; \beta_{1}, \beta_{2} ; x\right] \\
& +x \frac{\alpha_{2}-\beta_{1}}{\beta_{1}\left(\beta_{2}-\beta_{1}\right)}{ }_{2} F_{2}\left[\alpha_{1}, \alpha_{2} ; \beta_{1}+1, \beta_{2} ; x\right] \\
& +x \frac{\alpha_{2}-\beta_{2}}{\beta_{2}\left(\beta_{1}-\beta_{2}\right)}{ }_{2} F_{2}\left[\alpha_{1}, \alpha_{2} ; \beta_{1}, \beta_{2}+1 ; x\right],
\end{aligned}
$$

by the same as Theorem 7 calculating, we get the result (36).
Theorem 9. Let $X$ equipped with generalized logarithmic series distribution be defined in (6) with parameters $\theta$ and $\beta$, and $\mu_{-[l]}^{\prime}$ is the lth inverse factorial moment of $X$. Then the relation

$$
\begin{aligned}
& (l+1)(p-l-2) z \mu_{-[l+1]}^{\prime} \\
& \quad=[(p-2 l-2) z+l+1] \mu_{-[l]}^{\prime}-(1-z) \mu_{-[l-1]}^{\prime},
\end{aligned}
$$

holds for $l=2,3,4, \ldots, p=(n+1) \beta-n, n=0,1,2, \ldots$, $z=\theta /(1-\theta)^{1-\beta}$.

Proof. Using the identities (28) and (30), by the same as Theorem 7 calculating, we get the result (38).

\section{The Accurate Value for Inverse Moments of Some Discrete Distributions}

In this section, some accurate values for inverse moments of some discrete distributions can be obtained with their recurrence relations.

Theorem 10. Let $X$ be a generalized negative binomially distributed random variable with parameters $\theta$ and $\beta$, and probability mass function is defined in (3). Then the accurate value for inverse moment of first order is given by

$$
\begin{aligned}
& A E(X+A)^{-1}=\sum_{i=-1}^{A-3}(-1)^{i} \frac{(A+1-i)_{i} \cdot P_{1}}{(p-A-1)_{i+1} \cdot z^{i+1}}(1 \\
& -z)^{-p+1}+(-1)^{A-1} \frac{(3)_{A-2} \cdot P_{1}}{(p-A-1)_{A-1} \cdot z^{A-1}}{ }_{2} F_{1}[1, p ; \\
& 3 ; z]+\sum_{i=0}^{A-2}(-1)^{i} \\
& \quad \frac{(A+1-i)_{i}\{(A-i)+[p-(A+1-i) z]\}}{(p-A-1)_{i+1} \cdot z^{i+1}}{ }_{2} F_{1}[1 \\
& p ; 2 ; z], \quad
\end{aligned}
$$

where $p=m+(n+1) \beta-n, n=0,1,2, \ldots, z=\theta /(1-\theta)^{1-\beta}$, $P_{1}=P(X=1)=m \theta(1-\theta)^{m+\beta+1}$.

Proof. From (9), we have

$$
\begin{aligned}
A z(p-A-1) E(X+A)^{-1} & \\
= & A(1-A) E(X+A-1)^{-1}-P_{1}(1-z)^{-p+1} \\
& +[A+(p-A-1) z]_{2} F_{1}[1, p ; 2 ; z],
\end{aligned}
$$

and let $A=A-1$; we get

$$
\begin{aligned}
(A-1) & z(p-A) E(X+A-1)^{-1} \\
= & (A-1)(2-A) E(X+A-2)^{-1}-P_{1}(1-z)^{-p+1} \\
& \quad+[(A-1)+(p-A) z]_{2} F_{1}[1, p ; 2 ; z],
\end{aligned}
$$


and hence

$$
\begin{aligned}
A E(X+A)^{-1} & \frac{A(A-1)(A-2)}{(p-A-1)(p-A) \cdot z^{2}} E(X+A-2)^{-1} \\
& +\frac{A \cdot P_{1}(1-z)^{-p+1}}{(p-A-1)(p-A) \cdot z^{2}} \\
& -\frac{A[(A-1)+(p-A) z]}{(p-A-1)(p-A) \cdot z^{2}}{ }_{2} F_{1}[1, p ; 2 ; z] \\
& -\frac{P_{1}(1-z)^{-p+1}}{(p-A-1) \cdot z} \\
& +\frac{[A+(p-A-1) z]}{(p-A-1) \cdot z}{ }_{2} F_{1}[1, p ; 2 ; z],
\end{aligned}
$$

and so on; repeat the above steps; we can get

$$
\begin{aligned}
& A E(X+A)^{-1}=(-1)^{A-1} \frac{(2)_{A-1}}{(p-A-1)_{A-1} \cdot z^{A-1}} \\
& \cdot E(X+1)^{-1}+(-1)^{A-1} \\
& \cdot \frac{(3)_{A-2} \cdot P_{1}(1-z)^{-p+1}}{(p-A-1)_{A-1} \cdot z^{A-1}}+(-1)^{A-2} \\
& \cdot \frac{(4)_{A-3} \cdot P_{1}(1-z)^{-p+1}}{(p-A-1)_{A-2} \cdot z^{A-2}}+\cdots \\
& +(-1)^{2} \frac{(A)_{1} \cdot P_{1}(1-z)^{-p+1}}{(p-A-1)_{2} \cdot z^{2}}+(-1)^{1} \\
& \cdot \frac{(A+1)_{0} \cdot P_{1}(1-z)^{-p+1}}{(p-A-1)_{1} \cdot z}+(-1)^{A-2} \\
& \frac{(3)_{A-2}[2+(p-3) z]}{(p-A-1)_{A-1} \cdot z^{A-1}} \\
& \cdot{ }_{2} F_{1}[1, p ; 2 ; z]+(-1)^{A-3} \\
& \cdot \frac{(4)_{A-3}[3+(p-4) z]}{(p-A-1)_{A-2} \cdot z^{A-2}} \\
& \cdot{ }_{2} F_{1}[1, p ; 2 ; z]+\cdots+(-1)^{1} \\
& \frac{(A)_{1}[A-1+(p-A) z]}{(p-A-1)_{2} \cdot z^{2}} \\
& \cdot{ }_{2} F_{1}[1, p ; 2 ; z]+(-1)^{0} \\
& \frac{(A+1)_{0}[A+(p-A-1) z]}{(p-A-1)_{1} \cdot z} \\
& \cdot{ }_{2} F_{1}[1, p ; 2 ; z] \text {, }
\end{aligned}
$$

and, from (10), we have

$$
E(X+1)^{-1}=\frac{P_{1}}{2}{ }_{2} F_{1}[1, p ; 3 ; z],
$$

so we obtain the result (39).
Theorem 11. Let the random variable $X$ be equipped with a generalized Poisson distribution with parameters $\theta$ and $\lambda$, and probability mass function is defined in (4), and then the accurate value for inverse moment of first order is given by

$$
\begin{aligned}
A E(X+A)^{-1} & =\sum_{i=-1}^{A-3}(-1)^{i} \frac{(A-i)_{i+1} \cdot P_{1}}{z^{i+2}} \\
& +(-1)^{A-1} \frac{(3)_{A-2} \cdot P_{1}}{z^{A-1}}{ }_{1} F_{1}[1 ; 3 ; z] \\
& +\sum_{i=0}^{A-2}(-1)^{i} \frac{(A-i)_{i+1} \cdot P_{1}}{z^{i+1}}{ }_{1} F_{1}[1 ; 2 ; z],
\end{aligned}
$$

where $P_{1}=P(X=1)=\lambda e^{-\lambda(1+\theta)}, z=\lambda(1+(n+1) \theta) / e^{\lambda \theta}$, and $n=0,1,2, \ldots$.

The proof is the same as Theorem 10, omitted here.

Theorem 12. Let the random variable $X$ be equipped with a generalized poisson-negative-binomial distribution with parameters $\lambda, \theta$, and $\beta$, and probability mass function is defined in (5), and then the accurate value for inverse moment of first order is given by

$$
\begin{array}{rl}
A & E(X+A)^{-1}=\sum_{i=-1}^{A-3}(-1)^{i} \frac{(A-i)_{i+1} \cdot P_{1}}{(p-A-1)_{i+2} \cdot z^{i+2}}(1-z)^{-p+1} \\
& +(-1)^{A-1} \frac{(3)_{A-2} \cdot P_{1}}{(p-A-1)_{A-1} \cdot z^{A-1}}{ }_{2} F_{1}[1, p ; 3 ; z] \\
& +\sum_{i=0}^{A-2}(-1)^{i} \\
& +\frac{(A+1-i)_{i}\{(A-i)-[p-(A+1-i) z]\} \cdot P_{1}}{(p-A-1)_{i+1} \cdot z^{i+1}} F_{1}[1 \\
& p ; 2 ; z],
\end{array}
$$

where $P_{1}=P(X=1)=e^{-\lambda(1+\theta s)} \sum_{s=0}^{\infty}\left(\lambda^{s}(1+\theta s)^{s-1} / s !\right) m \theta(1-$ $\theta)^{m+\beta-1}, p=m+(n+1) \beta-n, n=0,1,2, \ldots$, and $z=\theta /(1-$ $\theta)^{1-\beta}$.

The proof is the same as Theorem 10, omitted here.

Theorem 13. Let $X$ be a generalized logarithmic distributed random variable with parameters $\theta$ and $\beta$, and probability mass function is defined in (6). Then the accurate value for inverse moment of first order is given by

$$
\begin{gathered}
A E(X+A)^{-1}=\sum_{i=-1}^{A-3}(-1)^{i} \frac{(A-i)_{i+1} \cdot P_{1}}{(p-A-1)_{i+2} \cdot z^{i+2}}(1-z)^{-p+1} \\
+(-1)^{A-1} \frac{(3)_{A-2} \cdot P_{1}}{(p-A-1)_{A-1} \cdot z^{A-1}}{ }_{2} F_{1}[1, p ; 3 ; z]
\end{gathered}
$$




$$
\begin{aligned}
& +\sum_{i=0}^{A-2}(-1)^{i} \\
& \cdot \frac{(A+1-i)_{i}\{(A-i)+[p-(A+1-i) z]\} \cdot P_{1}}{(p-A-1)_{i+1} \cdot z^{i+1}}{ }_{2} F_{1}[1, \\
& p ; 2 ; z],
\end{aligned}
$$

where $P_{1}=P(X=1)=\theta(1-\theta)^{\beta-1} /[-\log (1-\theta)], p=(n+$ 1) $\beta-n, n=0,1,2, \ldots$, and $z=\theta /(1-\theta)^{1-\beta}$.

The proof is the same as Theorem 10, omitted here.

\section{Conflict of Interests}

The authors declare that there is no conflict of interests regarding the publication of this paper.

\section{Acknowledgments}

The research is supported by the National Natural Science Foundation of China under Grant 11461050 and Natural Science Foundation of Inner Mongolia 2012MS0118.

\section{References}

[1] A. Kumar and P. C. Consul, "Negative moments of a modified power series distribution and bias of the maximum likelihood estimator," Communications in Statistics, vol. 8, no. 2, pp. 151166, 1979.

[2] M. T. Chao and W. E. Strawderman, "Negative moments of positive random variables," Journal of the American Statistical Association, vol. 67, no. 338, pp. 429-431, 1972.

[3] M. Ahmad and A. Saboor, "Properties of a newly defined hypergeometric power series function," Pakistan Journal of Statistics, vol. 25, no. 2, pp. 227-233, 2009.

[4] E. D. Rainville, Special Functions, Chelsea, New York, NY, USA, 1960. 


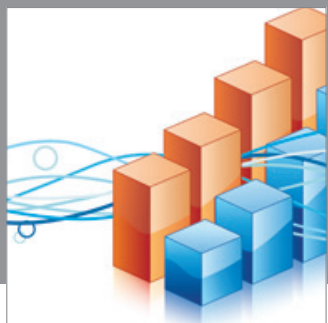

Advances in

Operations Research

mansans

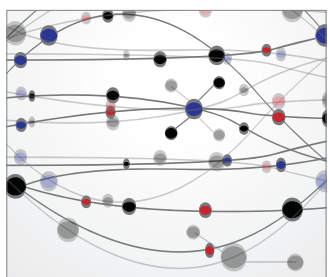

The Scientific World Journal
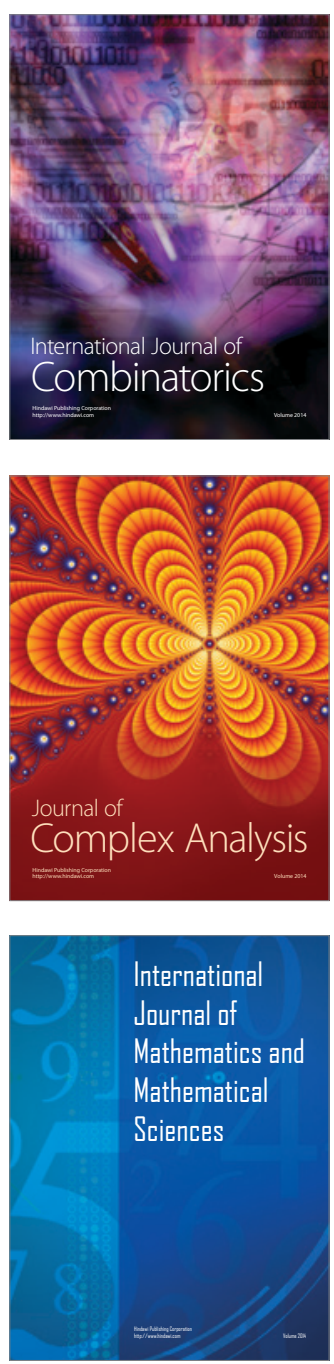
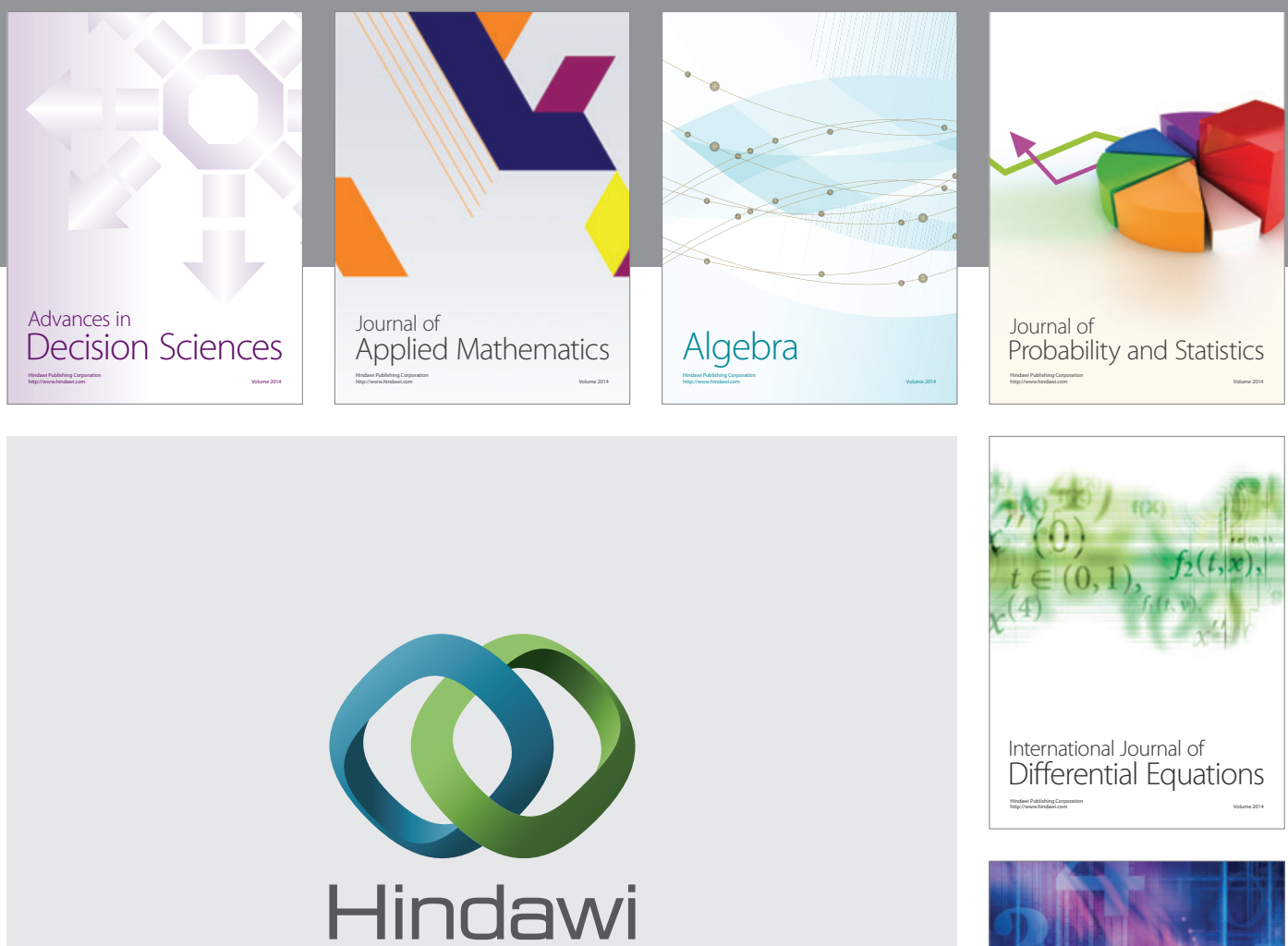

Submit your manuscripts at http://www.hindawi.com
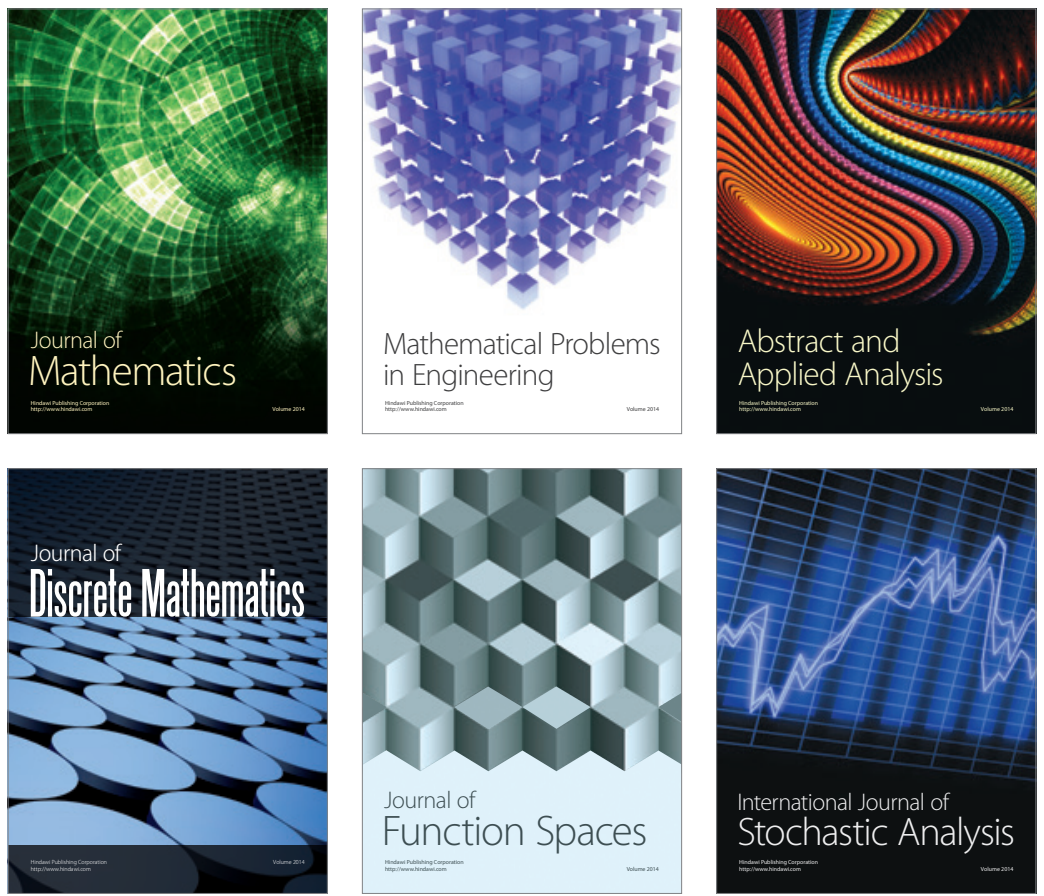

Journal of

Function Spaces

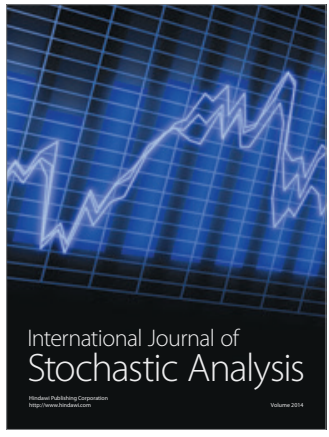

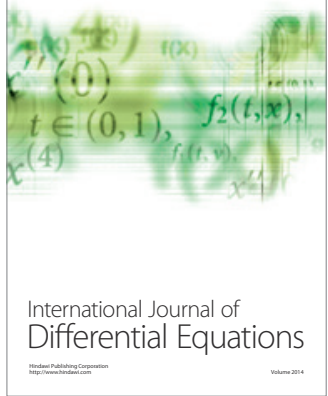
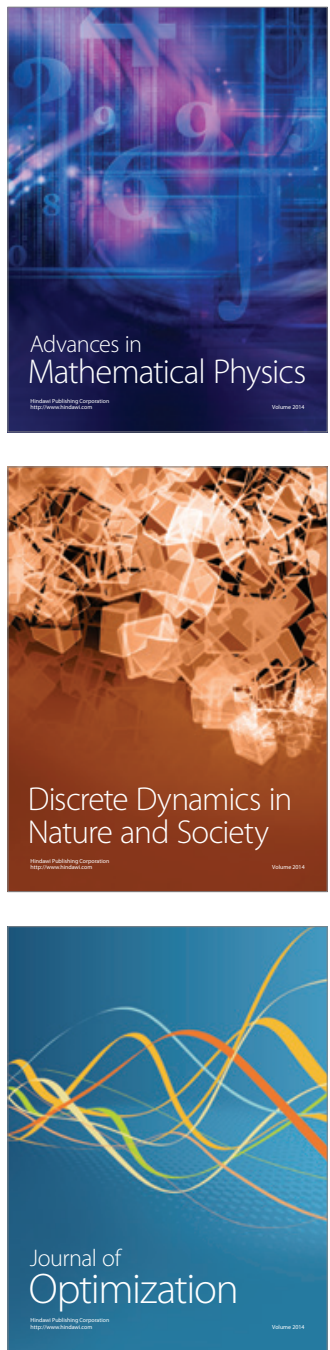